\title{
Valor terapéutico potencial de medicamentos en México: el caso de los antibióticos
}

Dolores Mino-León, ${ }^{1}$ Ixzel Hernández-Carvajal, ${ }^{2}$ Luis Peredo-Silva ${ }^{3}$ y Gabriel Búrbano-Martínez ${ }^{4}$

${ }^{1}$ Instituto Mexicano del Seguro Social, Centro Médico Nacional Siglo XXI, Hospital de Especialidades, Unidad de Investigación en Epidemiología Clínica, Ciudad de México; ${ }^{2}$ Universidad Autónoma de Morelos, Cuernavaca, Morelos; ${ }^{3}$ Universidad Nacional Autónoma de México, Facultad de Medicina, Ciudad de México; ${ }^{4}$ Investigador independiente. México

\section{Resumen}

Introducción: La relevancia de los medicamentos para la salud depende de su calidad, acceso y correcto uso. Objetivos: Determinar el valor terapéutico potencial de los antibióticos no incluidos en la lista de medicamentos esenciales (LME) de la Organización Mundial de la Salud pero que forman parte del Cuadro Básico de Medicamentos (CBM) de la Secretaría de Salud de México y categorizarlos por su valor intrínseco. Método: Análisis descriptivo de los antibióticos no incluidos en la LME de la Organización Mundial de la Salud 2013, revisión de la literatura para obtener evidencia de eficacia y seguridad y aplicación de escala de calidad y de valor intrínseco. Resultados: Se identificaron 452 resúmenes para 19 antibióticos, se eliminaron $56.9 \%$. In extenso se revisaron 195 ensayos clínicos; $37.9 \%$ fueron de calidad y se determinó valor intrínseco; 54 \% fueron estudios de superioridad y $46 \%$ de no inferioridad o equivalencia; $32 \%$ de los antibióticos fueron clasificados sin valor intrínseco y aproximadamente 50 \% fueron dudosos. Conclusión: Una elevada proporción de antibióticos del CBM tuvo valor intrínseco dudoso o nulo, lo que favorece su uso inapropiado, resistencia bacteriana y coloca a la población en riesgo.

PALABRAS CLAVE: Antibióticos. Valor terapéutico. Lista de medicamentos esenciales. Cuadro básico medicamentos.

\begin{abstract}
Introduction: The relevance of medications for health depends on their quality, accessibility and appropriate use. Objective: To determine the potential therapeutic value of antibiotics that are not included in the World Health Organization Essential Medicines List (EML) but that are part of the National Essential Medicines List (NEML) of the Mexican Ministry of Health, and categorize them according to their intrinsic value. Method: Descriptive analysis of antibiotics not included in the 2013 World Health Organization EML; literature review to obtain efficacy and safety evidence; and application of quality and intrinsic value scales. Results: Four hundred and fifty-two abstracts were identified for 19 antibiotics; $56.9 \%$ were excluded; 195 clinical trials were reviewed in full-text articles, out of which $37.9 \%$ were of good quality, and intrinsic value was determined; $54 \%$ were superiority studies, whereas $46 \%$ were non-inferiority or equivalence studies; $32 \%$ of the antibiotics were classified without intrinsic value and nearly $50 \%$ were inconclusive. Conclusion: An elevated proportion of antibiotics of the NEML had uncertain or no intrinsic value, which favors their inappropriate use, bacterial resistance and puts the population at risk.
\end{abstract}

KEY WORDS: Antibiotics. Therapeutic value. Essential Medicines List. National Essential Medicines List.

Correspondencia:

Dolores Mino-León

E-mail: minod_mx@yahoo.com
Fecha de recepción: 16-05-2018

Fecha de aceptación: 29-10-2018

DOI: 10.24875/GMM. 18004407
Gac Med Mex. 2019;155:15-19

Disponible en PubMed www.gacetamedicademexico.com 


\section{Introducción}

La relevancia de los medicamentos para la salud de una población depende de su calidad, acceso y correcto uso. A nivel global, la mitad de estos se prescribe, dispensa y consume de forma inadecuada. ${ }^{1}$ La Organización Mundial de la Salud (OMS) promueve el uso racional de medicamentos y recomienda la inclusión de políticas nacionales de medicamentos, ${ }^{2}$ ya que una vez que un medicamento se comercializa se requieren varios años para que sea reemplazado o para salir del mercado a partir de que se comprueba una relación beneficio/riesgo desfavorable. ${ }^{3-5}$

El mercado anual de antimicrobianos es de 960 millones de dólares, ocupa el segundo lugar en ventas y se consumen 950 millones de dosis. La compra y uso indiscriminado de este tipo de medicamentos ha favorecido la resistencia antimicrobiana. ${ }^{6}$ En Norteamérica se han reportado 23000 muertes/anuales por infecciones resistentes. ${ }^{7}$ La resistencia antimicrobiana ocasiona que un antibiótico pierda eficiencia, sea obsoleto y se retire del mercado. ${ }^{8}$

La OMS elaboró una lista de medicamentos esenciales (LME) para satisfacer la carga mundial de morbilidad. En México existen numerosos medicamentos con balance beneficio/riesgo desfavorable, ${ }^{5,9}$ el Cuadro Básico de Medicamentos (CBM) incluye antibióticos que no están en la LME de la OMS, por lo que es importante actualizarlo y evaluar la necesidad de retirar fármacos obsoletos o con relación beneficio/ riesgo nulo o dudoso y de costo inadecuado. ${ }^{10}$ Se ha planteado categorizar a los medicamentos de acuerdo con su valor terapéutico potencial o "valor intrínseco" (VI), que se basa en el análisis de su calidad terapéutica. ${ }^{10}$ Se ha utilizado la revisión de evidencia científica que al momento de la comercialización corresponde a ensayos clínicos (EC) y la escala de Jadad para evaluar la calidad metodológica de estos. ${ }^{11}$

Debido al impacto de los antibióticos sobre la salud pública, es importante determinar el valor terapéutico potencial de los que no están incluidos en la LME de la OMS, pero sí en el CBM de la Secretaría de Salud de México y categorizarlos de acuerdo con su VI.

\section{Método}

La LME 2013 de la OMS fue la referencia de los antibióticos que deberían estar incluidos en el CBM de la Secretaría de Salud (2014). Los antibióticos no incluidos en la LME de la OMS se analizaron y se les asignó su VI. Se revisaron las bases Medline (PubMed) y Cochrane, de enero de 1984 a diciembre de 2014, se incluyeron EC en inglés y español con el término clave "randomized controlled trials" combinado con el nombre genérico del antibiótico. Los resúmenes tenían que incluir las palabras "eficacia y/o seguridad" y la indicación tenía que ser "infección local y/o sistémica". Se eliminaron aquellos con indicación de profilaxis odontológica, uso tópico, erradicación de Helicobacter pylori, indicación diferente a infección y en los que no fue posible obtener el documento in extenso.

A los EC se les aplicaron los criterios de Jadad; aquellos que obtuvieron puntuación $\geq 3$ pasaron a la siguiente etapa. Los investigadores de forma individual y grupal revisaron los EC para categorizar el antibiótico de acuerdo con su VI:

- Elevado, medicamentos con eficacia demostrada en EC o cuya eficacia no ha sido demostrada en $E C$, pero cuyo uso se justifica en indicaciones definidas debido a que poseen efectos inmediatos y obvios.

- Relativo, medicamentos irracionales desde un punto de vista farmacológico y terapéutico porque, además de contener un principio activo de valor potencial elevado, contienen uno o más componentes químicos con eficacia terapéutica dudosa, cuya adición al preparado no se sustenta en ningún dato clínico obtenido en condiciones controladas.

- Dudoso, fármacos en los cuales existe controversia en la literatura internacional en relación con su eficacia a largo plazo.

- Sin VI, fármacos cuya eficacia no ha sido demostrada convincentemente en EC, pero sin descripción de efectos indeseables graves o frecuentes.

- Inaceptable, medicamentos que por su composición presentan una relación beneficio/riesgo claramente desfavorable en todas las circunstancias.

Se registró la participación de la industria farmacéutica y el objetivo del EC (superioridad, no inferioridad o equivalencia). Se realizó análisis descriptivo.

\section{Resultados}

Se analizaron 19 antibióticos, se identificaron 452 resúmenes, de los cuales se eliminaron 257. Se recuperaron 195 artículos in extenso a los que se les aplicó la escala de Jadad; únicamente se incluyeron $74(37.9 \%)$ con puntuación $\geq 3 ; 54 \%$ fueron de superioridad y $46 \%$ de no inferioridad o equivalencia. 
Tabla 1. Ensayos clínicos en los que se analizan antibióticos empleados en México, según su grupo terapéutico

\begin{tabular}{|c|c|c|c|c|c|c|c|c|c|c|c|}
\hline \multirow[t]{2}{*}{ Grupo } & \multirow{2}{*}{$\frac{\begin{array}{c}E C \\
(n=195)\end{array}}{n}$} & \multicolumn{2}{|c|}{$\begin{array}{c}\text { Jadad } \geq 3 \\
(n=74)\end{array}$} & \multicolumn{2}{|c|}{$\begin{array}{l}\text { Superioridad } \\
\quad(n=40)\end{array}$} & \multicolumn{2}{|c|}{$\begin{array}{l}\text { Superioridad relación } \\
\qquad \text { IF }(n=26)\end{array}$} & \multicolumn{2}{|c|}{$\begin{array}{l}\text { Equivalencia/No } \\
\text { inferioridad }(n=34)\end{array}$} & \multicolumn{2}{|c|}{$\begin{array}{l}\text { Equivalencia/No inferioridad } \\
\text { relación IF }(n=32)\end{array}$} \\
\hline & & $\mathrm{n}$ & $\%$ & $n$ & $\%$ & $\mathrm{n}$ & $\%$ & $\mathrm{n}$ & $\%$ & $\mathrm{n}$ & $\%$ \\
\hline Betalactámicos & 56 & 27 & 36.5 & 12 & 30 & 9 & 34.6 & 15 & 44.1 & 15 & 46.9 \\
\hline Cefalosporinas & 34 & 6 & 8.1 & 2 & 5 & 2 & 7.7 & 4 & 11.8 & 4 & 12.5 \\
\hline Macrólidos & 11 & 2 & 2.7 & 2 & 5 & 2 & 7.7 & 0 & 0 & 0 & 0 \\
\hline Aminoglucósidos & 18 & 11 & 14.9 & 8 & 20 & 3 & 11.5 & 3 & 8.8 & 2 & 6.2 \\
\hline Tetraciclinas & 13 & 2 & 2.7 & 2 & 5 & 1 & 3.8 & 0 & 0 & 0 & 0 \\
\hline Otros & 63 & 26 & 35.1 & 14 & 35 & 9 & 34.6 & 12 & 35.3 & 11 & 34.4 \\
\hline
\end{tabular}

$\mathrm{EC}=$ ensayo clínico, IF = Industria farmacéutica

El $65 \%$ de los de superioridad y $94.1 \%$ de no inferioridad o equivalencia reportaron relación con la industria farmacéutica.

El mayor porcentaje de los EC incluidos fueron betalactámicos (36.5\%). Todos los EC de macrólidos y tetraciclinas plantearon demostrar superioridad, mientras que la mayor proporción de EC de betalactámicos y otros fueron de equivalencia o no inferioridad. Respecto a la participación de la industria farmacéutica se observó mayor participación de esta en los EC de betalactámicos, cefalosporinas y otros (Tabla 1).

\section{Betalactámicos}

La combinación piperaciclina-tazobactam no superó al comparador y se clasificó con VI dudoso. Con meropenem, los resultados no reportaron superioridad y se le atribuyó VI dudoso. Ningún EC de dicloxacilina cumplió con criterios de calidad, se consideró sin VI. Un EC de superioridad de ertapenem confirmó esta, pero el resto reportó equivalencia, por lo que se atribuyó VI dudoso (Tabla 2).

\section{Cefalosporinas}

Ninguno EC con cefaclor, cefalotina y cefpiroma tuvo calidad metodológica, motivo por el cual se catalogaron sin VI. Los EC con cefepima y cefuroxima revelaron no inferioridad y se catalogaron con VI dudoso (Tabla 3).

\section{Macrólidos}

Roxitromicina no demostró superioridad y se catalogó con VI dudoso (Tabla 3).

\section{Aminoglucósidos}

La indicación de la tobramicina en todos los EC fue infección pulmonar en pacientes con fibrosis quística, para la cual demostró eficacia, motivo por el que se le atribuyó VI alto; la misma calificación se otorgó a neomicina (Tabla 4).

\section{Tetraciclinas}

La minociclina no superó a sus comparadores y encareció el tratamiento, por lo que se clasificó con VI dudoso. Los EC de oxitetraciclina/doxiciclina y tetraciclina no fueron de calidad y se catalogaron "sin VI" (Tabla 4).

\section{Otros}

Un EC de quinupristina/dalfopristina mostró eficacia similar al comparador, pero el comparador tuvo mayor riesgo de causar trombocitopenia, por lo que se le asignó VI alto. Los EC de tigeciclina demostraron que no es inferior por lo que su VI se consideró dudoso. Linezolid en general no fue superior y fue equivalente y no inferior respecto a su comparador; se le atribuyó un VI dudoso. La teicoplanina mostró ser eficaz y causar menos efectos secundarios comparada con la vancomicina; se le otorgó un VI alto (Tabla 5).

\section{Discusión}

La LME se sustenta en que "la selección cuidadosa de un número limitado de medicamentos resulta en una mejor calidad de atención, mejor uso y se favorece el gasto más eficiente de los recursos". Al elaborar una LME se debe considerar la prevalencia de las 
Tabla 2. Ensayos clínicos en los que se analizan betalactámicos que se emplean en México

\begin{tabular}{|c|c|c|c|c|c|}
\hline Nombre & Autor & Año & Calidad $^{\star}$ & Objetivo** & $\mathrm{IF}^{\star \star \star}$ \\
\hline \multicolumn{6}{|c|}{ Piperacilina-tazobactam } \\
\hline & Tan JS, et al. & 1993 & 4 & $\mathrm{~S}^{*}$ & No \\
\hline & Siami GA, et al. & 2001 & 4 & S & Sí \\
\hline & Siami FS, et al. & 2002 & 3 & S & Sí \\
\hline & Naber KG, et al. & 2002 & 3 & $\mathrm{NI}^{*}$ & Sí \\
\hline & Cornely $\mathrm{OA}$, et al. & 2004 & 3 & $\mathrm{NI}$ & Sí \\
\hline & Joshi M, et al. & 2006 & 4 & S & Sí \\
\hline & Schmitt DV, et al. & 2006 & 3 & S & Sí \\
\hline & Yamamoto Y, et al. & 2013 & 3 & S & No \\
\hline \multicolumn{6}{|c|}{ Meropenem } \\
\hline & Feld R, et al. & 2000 & 5 & S & Sí \\
\hline & Fabian TC, et al. & 2005 & 4 & $\mathrm{NI}$ & Sí \\
\hline & Lucasti C, et al. & 2013 & 5 & S & Sí \\
\hline \multicolumn{6}{|c|}{ Ertapenem } \\
\hline & Tomera KM, et al. & 2002 & 5 & $E^{*}$ & Sí \\
\hline & Ortiz-Ruiz G, et al. & 2002 & 4 & $E$ & Sí \\
\hline & Graham D, et al. & 2002 & 3 & $E$ & Sí \\
\hline & $\begin{array}{l}\text { Jiménez-Cruz F, } \\
\text { et al. }\end{array}$ & 2002 & 3 & $\mathrm{E}$ & Sí \\
\hline & Vetter N, et al. & 2002 & 4 & $\mathrm{E}$ & Sí \\
\hline & Roy S, et al. & 2003 & 5 & $E$ & Sí \\
\hline & Solomkin J, et al. & 2003 & 5 & $\mathrm{E}$ & Sí \\
\hline & Barboza E, et al. & 2003 & 3 & $\mathrm{E}$ & Sí \\
\hline & Gesser RM, et al. & 2004 & 5 & S & Sí \\
\hline & Lipsky BA, et al. & 2005 & 5 & S & Sí \\
\hline & Itani $\mathrm{K}$, et al. & 2006 & 5 & $\mathrm{NI}$ & Sí \\
\hline & Namias N, et al. & 2007 & 5 & $\mathrm{NI}$ & Sí \\
\hline & Arguedas A, et al. & 2009 & 3 & S & Sí \\
\hline & Park DW, et al. & 2012 & 4 & $\mathrm{E}$ & Sí \\
\hline & Catena F, et al. & 2013 & 4 & S & No \\
\hline & Solomkin JS, et al. & 2014 & 4 & S & Sí \\
\hline
\end{tabular}

${ }^{*}$ Superioridad (S), no inferioridad (NI), equivalencia (E)

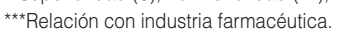

enfermedades, la evidencia científica y la relación costo-efectividad. ${ }^{12,13}$ Los resultados del estudio muestran una parte del problema en México, ya que una tercera parte de los antibióticos fue clasificada sin VI, lo que significa que la población está expuesta a un
Tabla 3. Ensayos clínicos en los que se analizan cefalosporinas y macrólidos que se emplean en México

\begin{tabular}{|c|c|c|c|c|c|}
\hline Nombre & Autor & Año & Calidad* & Objetivo** & IF*** \\
\hline \multicolumn{6}{|l|}{ Cefepima } \\
\hline & Raad II, et al. & 2003 & 3 & S & Sí \\
\hline & Yakovlev SV, et al. & 2006 & 5 & $\mathrm{NI}$ & Sí \\
\hline & Garbino J, et al. & 2007 & 5 & $\mathrm{NI}$ & Sí \\
\hline \multicolumn{6}{|c|}{ Cefuroxima } \\
\hline & Zervos MJ, et al. & 2003 & 4 & S & Sí \\
\hline & UpChurch J, et al. & 2006 & 5 & $\mathrm{NI}$ & Sí \\
\hline & Álvarez-Sala JL, et al. & 2006 & 3 & $\mathrm{NI}$ & Sí \\
\hline \multicolumn{6}{|c|}{ Roxitromicina } \\
\hline & Ortqvist A, et al. & 1996 & 3 & S & Sí \\
\hline & Hopstaken RM, et al. & 2002 & 5 & S & No \\
\hline
\end{tabular}

*Escala de Jadad.

${ }^{*}$ Superioridad (S), No inferioridad (NI), Equivalencia (E).

${ }^{* * *}$ Relación con industria farmacéutica.

Tabla 4. Ensayos clínicos en los que se analizan aminoglucósidos y tetraciclinas que se emplean en México

Nombre Autor Año Calidad* Objetivo** IF***

Tobramicina

$\begin{array}{lcccc}\text { Rodríguez JR, et al. } & 1985 & 3 & \text { S } & \text { Sí } \\ \text { Church DA, et al. } & 1997 & 3 & \text { E } & \text { Sí } \\ \text { Ramsey BW } & 1999 & 3 & \text { S } & \text { No } \\ \text { Bilton D, et al. } & 2006 & 3 & \text { S } & \text { Sí } \\ \text { Treggiari MM, et al. } & 2011 & 5 & \text { S } & \text { No } \\ \text { Trapnell BC, et al. } & 2012 & 3 & \text { S } & \text { No }\end{array}$

Neomicina

$\begin{array}{lllll}\text { van Balen FA, et al. } & 2003 & 5 & \text { S } & \text { No } \\ \text { Notivol R, et al. } & 2004 & 5 & \mathrm{NI} & \text { No } \\ \text { Roland PS, et al. } & 2008 & 4 & \mathrm{NI} & \text { Sí } \\ \text { Huttner B, et al. } & 2013 & 5 & \text { S } & \text { No } \\ \text { Pimentel M, et al. } & 2014 & 4 & \text { S } & \text { Sí }\end{array}$

Minociclina

\begin{tabular}{|c|c|c|c|c|}
\hline Ozolins M, et al. & 2004 & 4 & S & No \\
\hline Leyden J, et al. & 2006 & 3 & $S$ & Sí \\
\hline
\end{tabular}

riesgo innecesario. El hecho de que $38 \%$ de los EC tuvieran calidad metodológica satisfactoria dirige la atención a uno de los principios para seleccionar medicamentos: utilizar la mejor evidencia, ${ }^{13,14}$ y esto se 
Tabla 5. Ensayos clínicos en los que se analiza el grupo de antibióticos otros

Nombre Autor Año Calidad ${ }^{\star}$ Objetivo $^{\star *}$ IF $^{* * *}$

Quinupristina

Raad l, et al. $\quad 2004 \quad 3 \quad S \quad$ No

Tigeciclina

$\begin{array}{lllll}\text { Oliva ME, et al. } & 2005 & 4 & \mathrm{NI} & \text { Sí } \\ \text { Breedt J, et al. } & 2005 & 3 & \mathrm{NI} & \text { Sí } \\ \text { Fomin P, et al. } & 2005 & 5 & \mathrm{NI} & \text { Sí } \\ \text { Sacchidanand S, et al. } & 2005 & 4 & \mathrm{NI} & \text { Sí } \\ \text { Florescu I, et al. } & 2008 & 5 & \mathrm{~S} & \text { Sí } \\ \text { Tanaseanu C, et al. } & 2009 & 5 & \mathrm{NI} & \text { Sí } \\ \text { Bergallo C, et al. } & 2009 & 3 & \mathrm{NI} & \text { Sí } \\ \text { Freire AT, et al. } & 2010 & 3 & \mathrm{NI} & \text { Sí }\end{array}$

Linezolid

$\begin{array}{lllll}\text { Stevens DL, et al. } & 2000 & 4 & \text { S } & \text { Sí } \\ \text { Rubinstein E, et al. } & 2001 & 3 & \text { E } & \text { Sí } \\ \text { Wunderink RG, et al. } & 2003 & 3 & \text { S } & \text { Sí } \\ \text { Cepeda JA, et al. } & 2004 & 4 & \text { S } & \text { Sí } \\ \text { Jaksic B, et al. } & 2006 & 4 & \text { E } & \text { Sí } \\ \text { Lin DF, et al. } & 2008 & 3 & \text { Piloto } & \text { Sí } \\ \text { Wunderink RG, et al. } & 2012 & 5 & \text { NI } & \text { Sí } \\ \text { Moran GJ, et al. } & 2014 & 5 & \text { NI } & \text { Sí }\end{array}$

Teicoplanina

$\begin{array}{lllll}\text { Kureishi A, et al. } & 1991 & 5 & \text { S } & \text { Sí } \\ \text { Gillbert DN, et al. } & 1991 & 5 & \text { S } & \text { Sí } \\ \text { Chow AW, et al. } & 1993 & 4 & \text { S } & \text { No } \\ \text { Rolston KV, et al. } & 1994 & 5 & \text { S } & \text { No } \\ \text { Neville LO, et al. } & 1995 & 3 & \text { S } & \text { Sí } \\ \text { Liu CY, et al. } & 1996 & 3 & \text { S } & \text { Sí } \\ \text { Rolston KV, et al. } & 1999 & 4 & \text { E } & \text { No } \\ \text { Vazquez L, et al. } & 1999 & 3 & \text { S } & \text { No } \\ \text { D'Antonio D, et al. } & 2004 & 3 & \text { S } & \text { No }\end{array}$

*Escala de Jadad

${ }^{*}$ Superioridad (S), equivalencia (E), no inferioridad (NI)

${ }^{\star \star \star}$ Relación con industria farmacéutica. fortalece ya que casi la mitad tuvo VI dudoso, es decir, evidencia insuficiente. En la literatura se menciona que aproximadamente $70 \%$ de los medicamentos del mercado mundial es no esencial, es decir, no ofrece ninguna ventaja terapéutica en relación con los disponibles e, incluso, tiene mayor toxicidad ${ }^{14} \mathrm{o}$ incrementa los costos, ${ }^{15}$ como sucedió con la minociclina.

\section{Conclusión}

El hecho de que una proporción considerable de antibióticos tengan VI dudoso o nulo favorece su uso inapropiado, el desarrollo de resistencias bacterianas y coloca a la población en riesgo.

\section{Bibliografía}

1. World Health Organization. The world medicines situation 2004. Ginebra, Suiza: World Health Organization; 2004.

2. World Health Organization. The rational use of drugs. Report of the conference of experts, Nairobi, 25-29 November 1985. Ginebra, Suiza: World Health Organization; 1987.

3. Hoyle M. Historical lifetimes of drugs in England: application to value of information and cost-effectiveness analyses. Value Health. 2010;13: 885-892.

4. Diogene E, Figueras A. What public policies have been more effective in promoting rational prescription of drugs? J Epidemiol Community Health. 2011;65:87-88.

5. Rico-Alba I, Figueras A. The fuzzy line between needs, coverage, and excess in the Mexican formulary list: an example of qualitative market width analysis. Eur J Clin Pharmacol. 2013;69:949-956.

6. Dreser A, Wirtz VJ, Corbett KK, Echániz G. Uso de antibióticos en México: revisión de problemas y políticas. Salud Publica Mex. 2008; 50:S480-S487.

7. Floyd JS, Psaty BM. The potential risks of expedited approval of drugs for acute bacterial infections. JAMA Intern Med. 2014;174:1436-1437.

8. López-Navas A, García-Escribano Ráez N, Flores-Juberías Á, SuárezGea ML. Procedure for the marketing authorization of an antibacterial agent. Enferm Infecc Microbiol Clin. 2014;32:529-532.

9. Rico-Alba I, Figueras A. The evolution of reference drug lists and clinical practice guidelines in the public health system of a middle-income country. Br J Clin Pharmacol. 2014;78:410-421.

10. Laporte JR, Porta M, Capella D. Drug utilization studies: a tool for determining the effectiveness of drug use. Br J Clin Pharmacol. 1983;16: 301-304.

11. Jadad AR, Moore RA, Carroll D, Jenkinson C, Reynolds DJ, Gavaghan DJ, et al. Assessing the quality of reports of randomized clinical trials: is blinding necessary? Control Clin Trials. 1996;17:1-12.

12. World Health Organization. Selection of essential medicines. Ginebra, Suiza: World Health Organization; 2016.

13. Perumal-Pillay VA, Suleman F. Selection of essential medicines for South Africa: an analysis of in-depth interviews with national essential medicines list committee members. BMC Health Serv Res. 2017;17:17.

14. Management Sciences for Health. MSD-3: Managing access to medicines and health technologies. EE. UU.: Management Sciences for Health; 2012.

15. Ozolins M, Eady EA, Avery AJ, Cunliffe WJ, Po AL, O'Neill C, et al. Comparison of five antimicrobial regimens for treatment of mild to moderate inflammatory facial acne vulgaris in the community: randomised controlled trial. Lancet. 2004;364:2188-2195 